\title{
Medication Administered Domain
}

National Cancer Institute

\section{Source}

National Cancer Institute. Medication Administered Domain. NCI Thesaurus. Code C154637.

Records of medications administered to patients by healthcare providers. These administrations may take place in any setting, including inpatient, outpatient or home health encounters. 